\title{
Physical Activity and Sports in Dementia
}

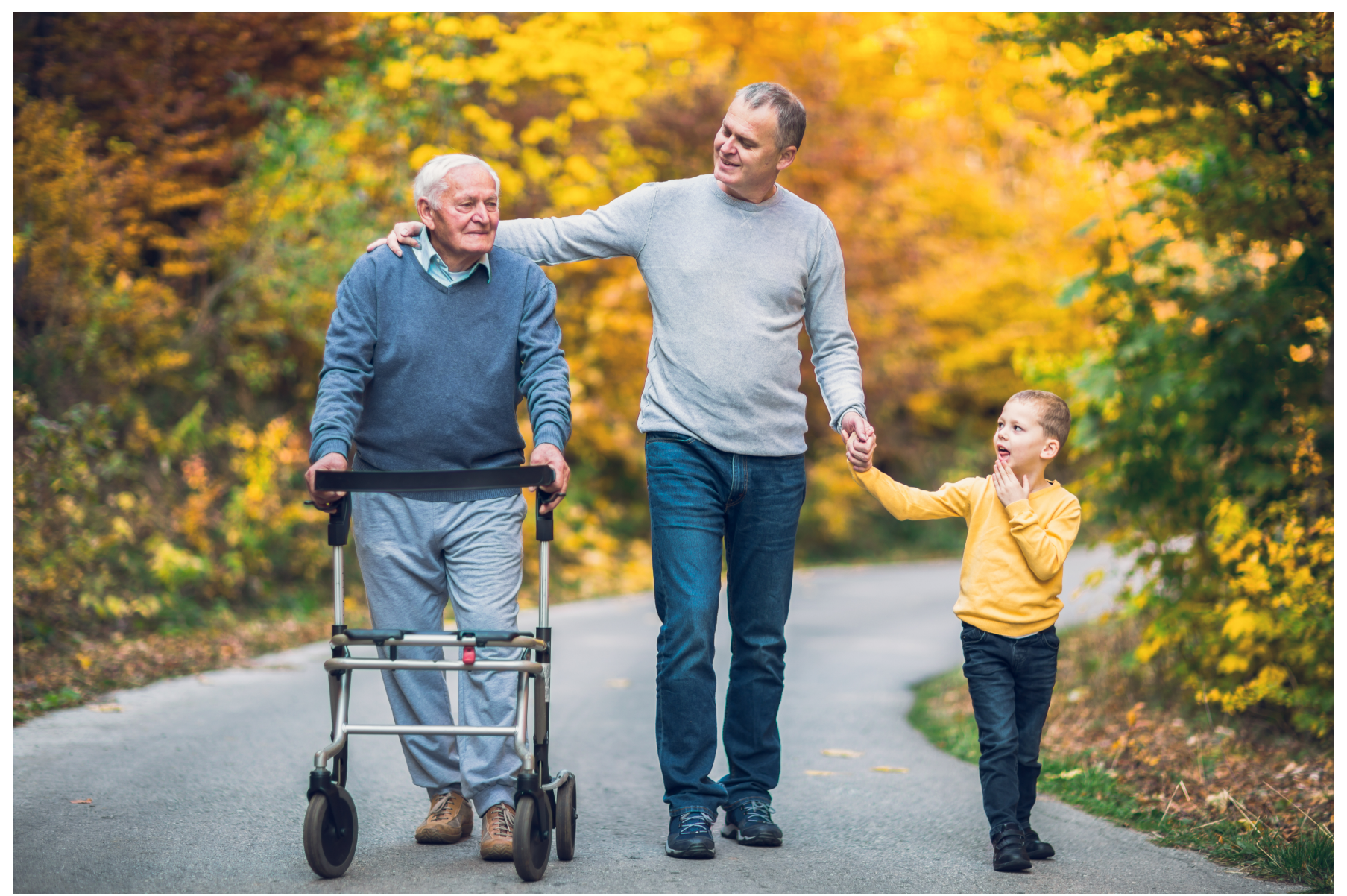

Körperliche Aktivität und sportliche Bewegung bei Demenz

Ngamsri $\mathrm{T}^{1}$, Claussen $\mathrm{MC}^{2}$, Hemmeter $\mathrm{UM}^{1}$

${ }^{1}$ Psychiatrie St. Gallen Nord

${ }^{2}$ Klinik für Psychiatrie, Psychotherapie und Psychosomatik, Psychiatrische Universitätsklinik Zürich und Privatklinik Wyss AG

\section{Abstract}

There is a continuous increase in dementia partly due to increasing life-expectance. Currently there are no 
causal therapies for dementia, neither for dementias with vascular etiology nor for neurodegenerative dementias such as Alzheimer's disease.

Main risk factors for the development of dementia are low physical activity, hypertonia and diabetes mellitus. Physical activity has shown to exert beneficial effects on cardiovascular and metabolic risk factors und is closely connected with cognitive disturbance and the development and the course of dementia.

Therefore, the implementation of physical activity in preventive and therapeutic strategies of dementia is recommended.

\section{Zusammenfassung}

Demenzen nehmen nicht zuletzt wegen der höheren Lebenserwartung stetig zu. Bisher gibt es keine ursächliche Therapie der Demenzen, weder für Demenz mit vaskulärer noch für Demenzen mit neurodegenerativer Genese. Als wesentliche Risikofaktoren für die Entwicklung und den Verlauf von Demenzen wurden u.a. mangelnde Bewegung, Hypertonie und Diabetes mellitus identifiziert.

Körperliche Aktivität hat günstige Effekte auf die kardiovaskulären und metabolischen Risikofaktoren und steht auch mit dem Auftreten kognitiver Störungen sowie der Entwicklung und dem Verlauf von Demenzen in engem Zusammenhang.

Der gezielte Einsatz von körperlicher Aktivität im Sinne von sportlicher Bewegung kann daher sowohl präventiv wie auch therapeutisch bei Demenzen genutzt werden.

\section{Einleitung}

Wie die Sportmedizin untersucht die Sportpsychiatrie und -psychotherapie den Einfluss von Bewegung, Training und Sport sowie des Bewegungsmangels auf den gesunden und kranken Menschen jeder Altersstufe mit dem Ziel, die gewonnenen Erkenntnisse in der Diagnostik und Therapie, wie Prävention und Rehabilitation sowie zum Wohle des Sports einzusetzen. Sportler aller Leistungsklassen stehen dabei genauso im Fokus der Sportpsychiatrie und -psychotherapie. Mit den therapeutischen und rehabilitativen Möglichkeiten von Sport sowie der Vorbeugung, Erkennung und Behandlung und Rehabilitation von Sportverletzungen und Sportschäden beschäftigt sich die Sportpsychiatrie und -psychotherapie ebenso, immer bezogen auf die Aspekte, die das medizinische Wissen des Fachs Psychiatrie und Psychotherapie betreffen. Im folgenden Artikel soll am Beispiel der Demenz die Bedeutung von körperlicher Aktivität und sportlicher Bewegung in der Psychiatrie und Psychotherapie, als ein Aspekt der Sportpsychiatrie und psychotherapie - und der Sportmedizin - vorgestellt und diskutiert werden.

Im Jahr 2019 lebten in der Schweiz ca. 154700 Menschen mit einer Demenz. Im Jahr 2040 werden es voraussichtlich 300000 Demenzerkrankte Menschen sein [1]. Weltweit lebten im Jahr 201035.6 Millionen Menschen mit einer diagnostizierten Demenz mit deutlich steigender Tendenz [2].

Es zeigt sich zunehmend, dass neben dem hohen Alter und genetischen Faktoren auch vaskuläre Risikofaktoren und psychosoziale Faktoren eine wichtige Rolle bei der Demenzentstehung spielen [3]. Es wird davon ausgegangen, dass bei etwa einem Drittel aller Patienten mit Alzheimer-Demenz (AD) sieben modifizierbare Risikofaktoren die Entwicklung und den Verlauf wesentlich beeinflussen. Diese sind Diabetes mellitus, Hypertonie und Adipositas im mittleren Lebensalter sowie Bewegungsmangel, Depressionen, Rauchen und ein geringer Bildungsstand [4]. 
Für ca. 30\% der Demenzfälle sind sieben modifizierbare Risikofaktoren verantwortlich

- Körperliche Inaktivität

- Geringer Bildungsstand

- Rauchen

- Hypertonie im mittleren Lebensalter

- Adipositas im mittleren Lebensalter

- Diabetes mellitus

- Depression

Mehrere Studien zeigen, dass körperliche Aktivität zur Prävention von Demenzerkrankungen eingesetzt werden kann, indem der kognitive Abbau und das Eintreten einer AD verzögert wird [5,6]. Körperliche Aktivität ist im Vergleich zu anderen Präventionsmassnahmen einfach durchzuführen und bei regelmässiger Durchführung sehr effektiv.

Durch körperliche Aktivität wird auch das Risiko für Myokardinfarkte, Schlaganfälle und Diabetes mellitus reduziert. Dadurch kann sekundär auch das Risiko für eine Demenzentwicklung reduziert bzw. deren Verlauf verzögert werden [7].

Diese Zusammenhänge zwischen körperlicher Aktivität, Kognition und Demenz weisen auf die mögliche präventive Rolle der Bewegung für die Entwicklung einer Demenz hin. Die Prävention kann somit aus einer multimodalen Strategie bestehen, die insgesamt die Anpassung des Lebensstils in Bezug auf Ernährung, körperliche und geistige Aktivität zum Inhalt hat [8].

\section{Effekte von sportlicher Bewegung auf kardiovaskuläre Faktoren und mentale}

\section{Gesundheit}

Wie bereits zuvor beschrieben, hat körperliche Aktivität umfassende positive Auswirkungen auf die allgemeine Gesundheit.

In Bezug auf den Zusammenhang zwischen körperlicher Aktivität und sowohl kardiovaskulären Risikofaktoren im Allgemeinen als auch chronische Herzerkrankungen im Besonderen scheint eine DosisWirkungs-Kurve zu bestehen, indem mehr körperliche Bewegung auch eine bessere Gesundheit zur Folge hat. Weitere Studien zeigen positive Auswirkungen von körperlicher Aktivität auf die arterielle Hypertonie wie auch auf Diabetes mellitus. Die hier diskutierten biologischen Mechanismen und Zusammenhänge (s.u.) sind plausibel und werden von diversen klinischen und Beobachtungsstudien unterstützt [7]. Für Diabetes mellitus gibt es Leitlinienempfehlungen zur Prävention und Therapie durch Steigerung von körperlicher Aktivität. Diese spielt auch bei der Steigerung der Insulinresistenz und der Gewichtsreduktion eine wichtige Rolle [9].

Neben dem positiven Einfluss auf die kardiovaskulären Risikofaktoren und eine diabetogene Stoffwechsellage hat sportliche Bewegung auch einen Einfluss auf die mentale und psychische Gesundheit. Es wird in verschiedenen Studien von einer Linderung depressiver Symptome und Angstsymptome sowie einer aufhellenden Wirkung auf die Stimmung berichtet [7,10]. 


\section{Generelle Effekte von Bewegung auf kognitive Funktionen bei verschiedenen}

\section{chronischen Hirnerkrankungen}

In einer aktuellen Meta-Analyse von Dauwan et al. (2019) wird diesem Zusammenhang nachgegangen. Es konnten hier Zusammenhänge und Effekte auf die unterschiedlichen kognitiven Funktionen bei Patienten mit verschiedenen chronischen Hirnerkrankungen (u.a. mit Alzheimer-Demenz [AD], HuntingtonKrankheit [HD], Parkinson-Erkrankung [PD]) aufgezeigt werden. In der Analyse von 21 Studien (an 1313 Probanden mit AD, HD, PD) wurden eine signifikant bessere Aufmerksamkeit und ein besseres Arbeitsgedächtnis durch körperliche Aktivität gezeigt.

In derselben Meta-Analyse zeigte sich in 14 Studien (an 977 Probanden mit AD, HD, PD) auch ein signifikant positiver Zusammenhang auf die exekutiven Funktionen. 12 Studien (an 994 Probanden mit AD, HD, PD) zeigten einen positiven Effekt auf Gedächtnisleistungen, welcher hauptsächlich durch strukturiert und konsequent durchgeführte Aerobic- Übungen zustande kam.

Einen geringeren, aber signifikanten Effekt zeigte körperliche Aktivität bei der psychomotorischen Geschwindigkeit (16 Studien, 896 Probanden mit AD, HD, PD). Es wurde ein klarer Trend zur Verbesserung der globalen Kognition durch körperliches Training nachgewiesen. Lediglich für die Wortflüssigkeit (verbal fluency) ergab sich kein signifikanter Zusammenhang [10].

\section{Zusammenhang von Bewegung auf das Demenzrisiko im Speziellen}

Stephen et al. (2017) zeigen in ihrer Meta-Analyse von 24 Studien eine Risikoreduktion für AD durch körperliche Aktivität in der Freizeit. Diese Risikoreduktion war jedoch kaum nachweisbar bei körperlicher Aktivität im Berufsalltag. Es wird daher angenommen, dass Bewegung im beruflichen Alltag allein nicht genügt.

In einer Arbeit von Rovio et al. (2007) konnte bei berufsbedingter körperlicher Aktivität sogar ein höheres Risiko für AD nachgewiesen werden. Berufsbedingte körperliche Aktivität ist aber auch assoziiert mit einem niedrigeren Bildungsstand, einem niedrigeren sozioökonomischen Status sowie einer geringeren kognitiven Aktivität. Diese Faktoren sind wiederum nachweislich Risikofaktoren für AD und Demenz allgemein, wodurch sich dieses Resultat erklärt. Zudem zeigt sich ein umgekehrt proportionaler Zusammenhang von körperlicher Aktivität in Beruf und Freizeit. D.h. beruflich bedingte sitzende Tätigkeiten sind eher mit körperlicher Aktivität in der Freizeit verbunden [11], die dann auch einen präventiven Effekt haben. Hinzu kommt, dass körperliche Aktivität in der Freizeit eher mit sozialer und kognitiver Stimulation als weitere protektive Faktoren für die AD-Entwicklung assoziiert sind [12]. Es gibt auch einige wenige Studien, die keinen Zusammenhang von körperlicher Aktivität mit dem Demenzrisiko zeigen. Diese weisen jedoch methodische Mängel auf, indem sie nicht zwischen körperlicher Aktivität in der Freizeit, im Beruf und im Haushalt differenzieren oder vage, nicht klar definierte Begriffe wie «körperliche Aktivität im Allgemeinen» für die Intervention beschreiben [13].

Die überwiegende Mehrzahl der Arbeiten weist aber auf einen Zusammenhang zwischen körperlicher Aktivität und reduziertem Demenzrisiko sowie besserer kognitiver Leistungsfähigkeit hin (Stephen et al. 2017).

In der Metaanalyse von Stephen R. und Kollegen (2107) zeigten alle Studien der körperlichen Aktivität in der Freizeit im mittleren Lebensalter einen klaren günstigen Effekt zur Reduktion des AD-Risikos. Auch die 
Steigerung körperlicher Aktivität im Lebensverlauf kann mit einem reduzierten Risiko zur ADEntwicklung verbunden sein. In einer bevölkerungs-basierten Studie konnten protektive Effekte gegen Demenz bei längerer, Freizeit-abhängiger, körperlicher Aktivität ab dem mittleren Lebensalter, aber auch bei Steigerung des Aktivitätsniveaus im späteren Lebensabschnitt nachgewiesen werden [14].

\section{Körperliche Aktivität als therapeutische Intervention bei Demenzerkrankungen}

Es gibt Hinweise, dass Bewegung eine sinnvolle therapeutische Intervention zur Behandlung von Patienten mit einer Alzheimer-Demenz sowie bei Patienten mit einer beginnenden Alzheimer-Demenz sein kann [15]. Diese konnte eine teilweise Verbesserung der kognitiven Funktionen erzielen, vor allem aber eine Verbesserung der neuropsychiatrischen Symptome und eine Verlangsamung des Rückgangs der Selbstständigkeit (Aktivitäten des täglichen Lebens) [16]. Dasselbe gilt auch für die Behandlung von Patienten mit vaskulärer Demenz, jedoch fehlen hierzu noch aussagekräftige Studien [17].

\section{Frequenz und Dauer der körperlichen Aktivität}

Aus den bisher durchgeführten Studien, lässt sich keine klare Empfehlung ableiten, in welcher Intensität, d.h. in welcher Frequenz und Dauer körperliche Aktivität durchgeführt werden muss, um eine gute präventive Wirkung oder einen Therapieerfolg (z.B. auf behaviorale und psychische Symptome der Demenz, BPSD) zu erzielen. Manche Autoren beziehen ihre Empfehlung zur Durchführung der körperlichen Aktivität im Sinne von sportlicher Bewegung bei Demenz auf bestehende Empfehlungen zur Gesundheitsförderung. Solche Empfehlungen können auch das AD-Risiko senken [18].

Trotz der Vorbehalte zu einer Empfehlung von Dauer und Art der körperlichen Aktivität, kann aus einer Arbeit von Thompson et al. (2003) der Gesamtenergieverbrauch pro Tag abgeleitet werden, der mit der Prävention von kardiovaskulärem Risiko in Verbindung gebracht wird, nicht aber die Art der Aktivität [19].

Da es nachgewiesen ist, dass die Prävention und Verbesserung der kardiovaskulären Risikofaktoren (wie z.B. arterielle Hypertonie und Diabetes mellitus) eine Risikoreduktion der Entwicklung von Demenzerkrankungen hat [4], kann abgeleitet werden, dass die Empfehlungen für sportliche Bewegung zur Verbesserung dieser kardiovaskulären Risikofaktoren sekundär auch einen Effekt auf die Risikoreduktion der Demenz haben können.

Zudem gibt es auch Empfehlungen hierzu zur Wirksamkeit von sportlicher Bewegung, z.B. bei Diabetes, an denen man sich orientieren kann.

Es wird empfohlen, ein regelmässiges aerobes Training durchzuführen an mindestens drei Tagen pro Woche (Pausen zwischen den Trainingseinheiten nicht länger als zwei aufeinanderfolgende Tage aufgrund des nachlassenden positiven Effektes auf die Insulinaktivität). Bezüglich der Intensität wird eine mindestens moderate Intensität empfohlen und eine Dauer von mindestens 150 Minuten pro Woche. Ausdauerübungen können schnelles Gehen, Nordic Walking, Laufen (Joggen), Schwimmen, Radfahren oder auch Berg- bzw. Skiwandern sein [20]. 


\section{Neurobiologische Befunde mit Zusammenhang zur Wirkung von körperlicher}

\section{Aktivität bei Demenz}

Die genauen zugrunde liegenden Mechanismen des positiven Effektes von körperlicher Aktivität auf die Kognition und das verringerte Demenzrisiko sind noch weitgehend ungeklärt. Eine mögliche Ursache für die beobachtete Reduktion des Demenzrisikos durch körperliche Aktivität ist die Verbesserung der zerebralen Perfusion und die Stimulation von Neurogenese, Synaptogenese und Angiogenese [21]. Dadurch kann der Nervenzellverlust reduziert werden, wodurch das Gehirnvolumen der AD-anfälligen Regionen (z.B. Hippokampusvolumen) länger erhalten bleibt [22]. Zudem konnte gezeigt werden, dass körperliche Aktivität auch einen positiven Einfluss auf die ADtypischen neuropathologischen Prozesse wie die beta-Amyloid-Akkumulation und Tau-Phosphorylierung hat, wobei unklar ist, durch welche zugrunde liegenden neurobiologischen und neuropathologischen Vorgänge diese Beobachtung bewirkt wird [23]. Aktuelle Tiermodell-Versuche weisen dem BDNF (brainderived-neurotrophic-factor) eine Schlüsselrolle zu. Diese Hypothese stützt sich auf Untersuchungen, die zeigen konnten, dass die körperliche Aktivität die Synthese und Akkumulation von neuroaktiven Metaboliten wie Myokinen und Ketonkörpern in der Peripherie und im Hippokampus fördert, um dann auch die Expression von BDNF zu fördern [24]. Das BDNF ist ein Neurotrophin mit verschiedenen Eigenschaften, u.a. zur Förderung des neuralen Überlebens und synaptischer Integrität. Sein Einfluss auf die Energieübertragung stellt eine wichtige Funktion für die Assoziation von vaskulären und metabolischen Faktoren zur körperlichen Aktivität dar. Im Mausmodell zeigte ein erhöhtes körperliches Training eine höhere BDNF-Expression, die gleichzeitig mit einem verbesserten räumlichen Gedächtnis assoziiert war. Durch Hemmung der BDNF-Übertragung wurden diese Effekte wieder aufgehoben [25]. Dem BDNF kommt somit bei der Assoziation zwischen körperlicher Aktivität und Demenzrisiko eine Schlüsselrolle zu, die weiter aufgeklärt werden muss.

\section{Zusammenfassung und Fazit}

Während die Sportpsychiatrie und -psychotherapie innerhalb der Psychiatrie und Psychotherapie zunehmend wahrgenommen wird, muss es eine der zentralen Aufgaben der nächsten Zeit sein, sich ebenso als Teilgebiet der Sportmedizin zu etablieren.

Bezogen auf die Aspekte der Sportpsychiatrie und -psychotherapie, die hier vorgestellt und diskutiert wurden, ist festzuhalten, dass es deutliche Hinweise auf einen Zusammenhang zwischen körperlicher Aktivität und der Entstehung sowie des Verlaufs von Demenzen gibt. Ob körperliche Aktivität einen direkten Einfluss darauf hat oder über eine günstige Beeinflussung primärer Risikofaktoren wie Hypertonie und Diabetes mellitus bzw. metabolisches Syndrom indirekt dazu beiträgt, kann zum gegenwärtigen Zeitpunkt nicht sicher beantwortet werden.

Aus den bisher vorliegenden Befunden lässt sich ableiten, dass eine regelmässige aerobe Aktivität in der Freizeit an mindestens drei Tagen pro Woche mit einer moderaten Intensität und einer Dauer von mindestens 150 Minuten pro Woche die Entwicklung und den Verlauf einer Demenz günstig beeinflussen kann. Ausdauerübungen wie schnelles Gehen, Nordic Walking, Laufen (Joggen), Schwimmen, Radfahren oder auch Berg- bzw. Skiwandern können hier empfohlen werden.

Körperliche Aktivität bzw. sportliche Bewegung stellt jedoch nur einen Teilaspekt der Prävention der 
Demenz dar, da eine Modifikation des Lebensstils insgesamt mit Anpassung der Ernährung, geistiger Aktivierung und Beeinflussung der vaskulären Risikofaktoren den stärksten Effekt auf die Reduktion und das verzögerte Auftreten von Demenz-Erkrankungen hat.

\section{Korrespondenzadresse}

Theofanis Ngamsri

Dr. med., Oberarzt

Psychiatrie St. Gallen Nord

theofanis.ngamsri@psgn.ch

+41719131219

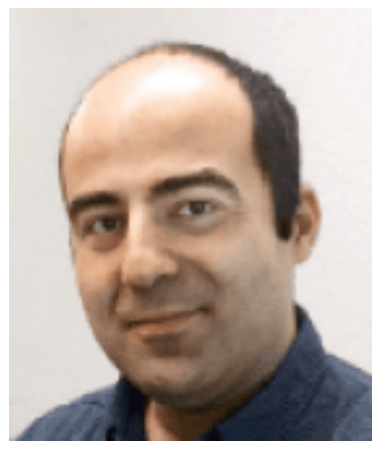

口Malte Christian Claussen

Dr. med.

Ärztlicher Leiter Sportpsychiatrie

und -psychotherapie,

Psychiatrische Universitätsklinik Zürich

und Privatklinik Wyss AG

Präsident Schweizerische Gesellschaft

für Sportpsychiatrie und

-psychotherapie (SGSPP)

malte.claussen@puk.zh.ch

+41443842621

Ulrich Michael Hemmeter

PD Dr. med. Dr. phil.

Chefarzt

Psychiatrie St. Gallen Nord

ulrich.hemmeter@psgn.ch

+41719131202 ?
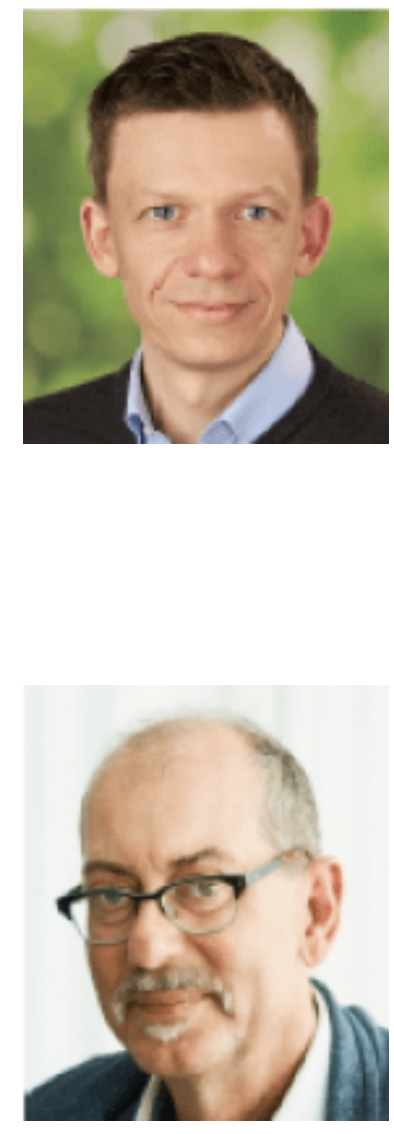
Münchenbuchsee gegründet. Der Zweck der SGSPP ist die Förderung der Sportpsychiatrie und psychotherapie über die Lebensspanne in der Schweiz, im Leistungssport und in der Allgemeinbevölkerung. Eine aktive Teilnahme am weiteren Aufbau und der Ausrichtung der SGSPP ist sehr willkommen. Weitere Informationen zur SGSPP und Mitgliedschaft finden sich auf der SGSPP-Homepage: http://www.sgspp.ch.

\section{Jahrestagung der SGSPP}

Die 1. Jahrestagung der Schweizerischen Gesellschaft für Sportpsychiatrie und -psychotherapie (SGSPP) wird am

31. Januar 2020 in der Psychiatrischen Universitätsklinik Zürich stattfinden. Vertreter aller deutschsprachigen sportpsychiatrischen und -psychotherapeutischen Initiativen haben ihr Kommen zugesagt. Eine Anmeldung ist nicht erforderlich und die Teilnahme ist kostenlos. Weitere Informationen auf: http://www.sgspp.ch.

\section{Referenzen}

1. Schweiz, A., Demenz in der Schweiz 2019: Zahlen und Fakten. 2019.

2. Wimo, A., et al., The worldwide economic impact of dementia 2010. Alzheimers Dement, 2013. 9(1):p.1-11.e3.

3. Qiu, C., M. Kivipelto, and E. von Strauss, Epidemiology of Alzheimer's disease: occurrence, determinants, and strategies toward intervention. Dialogues Clin Neurosci, 2009. 11(2):p.111-28.

4. Norton, S., et al., Potential for primary prevention of Alzheimer's disease: an analysis of populationbased data. Lancet Neurol, 2014. 13(8):p.788-94.

5. Tan, Z.S., et al., Physical Activity, Brain Volume, and Dementia Risk: The Framingham Study. J Gerontol A Biol Sci Med Sci, 2017. 72(6):p.789-795.

6. $\mathrm{Xu}, \mathrm{W}$., et al., Leisure time physical activity and dementia risk: a dose-response meta-analysis of prospective studies. BMJ Open, 2017. 7(10):p.e014706.

7. Services, U.S.D.o.H.a.H., U.S. Department of Health and Human Services. Physical Activity and Health: A Report of the Surgeon General. Atlanta, GA: U.S. Department of Health and Human Services, Centers for Disease Control and Prevention, National Center for Chronic Disease Prevention and Health Promotion. 1996.

8. Ngandu, T., et al., A 2 year multidomain intervention of diet, exercise, cognitive training, and vascular risk monitoring versus control to prevent cognitive decline in at-risk elderly people (FINGER): a randomised controlled trial. Lancet, 2015. 385(9984):p.2255-63.

9. Standards of medical care in diabetes-2012. Diabetes Care, 2012. 35 Suppl 1:p.S11-63.

10. Dauwan, M., et al., Physical exercise improves quality of life, depressive symptoms, and cognition across chronic brain disorders: a transdiagnostic systematic review and meta-analysis of randomized controlled trials. J Neurol, 2019.

11. Rovio, S., et al., Work-related physical activity and the risk of dementia and Alzheimer's disease. Int J Geriatr Psychiatry, 2007. 22(9):

12. p.874-82.

13. Verghese, J., et al., Leisure activities and the risk of dementia in the elderly. N Engl J Med, 2003. 348(25):p.2508-16.

14. Stephen, R., et al., Physical Activity and Alzheimer's Disease: A Systematic Review. J Gerontol A Biol Sci Med Sci, 2017. 72(6):p.733-739.

15. Tolppanen, A.M., et al., Leisure-time physical activity from mid- to late life, body mass index, and risk 
of dementia. Alzheimers Dement, 2015. 11(4):p.434-443.e6.

16. Maliszewska-Cyna, E., et al., The Benefits of Exercise and Metabolic Interventions for the Prevention and Early Treatment of Alzheimer's Disease. Curr Alzheimer Res, 2017. 14(1):p.47-60.

17. Cass, S.P., Alzheimer's Disease and Exercise: A Literature Review. Curr Sports Med Rep, 2017. 16(1):p.19-22.

18. Mori, E., [How Treatable is Vascular Dementia?]. Brain Nerve, 2016. 68(4):p.441-50.

19. Verdelho, A., et al., Physical activity prevents progression for cognitive impairment and vascular dementia: results from the LADIS (Leukoaraiosis and Disability) study. Stroke, 2012. 43(12):p.3331-5.

20. Thompson, P.D., et al., Exercise and physical activity in the prevention and treatment of atherosclerotic cardiovascular disease: a statement from the Council on Clinical Cardiology (Subcommittee on Exercise, Rehabilitation, and Prevention) and the Council on Nutrition, Physical Activity, and Metabolism (Subcommittee on Physical Activity). Circulation, 2003. 107(24):p.3109-16.

21. Leitlinie, N.V., Therapie des Typ-2-Diabetes Langfassung 2013. Programm für Nationale Versorgungs Leitlinien, 2013.

22. Holsinger, R.M.D., Translation of low-risk dementia-associated interventions into practice - a call to action. Healthy Aging Research 4:30. doi:10.12715/har.2015.4.30 2015.

23. Erickson, K.I., et al., Exercise training increases size of hippocampus and improves memory. Proc Natl Acad Sci U S A, 2011. 108(7):

24. p.3017-22.

25. Garcia-Mesa, Y., et al., Physical exercise protects against Alzheimer’s disease in 3xTg-AD mice. J Alzheimers Dis, 2011. 24(3):p.421-54.

26. Mattson, M.P., et al., Neurotrophic factors attenuate glutamate-induced accumulation of peroxides, elevation of intracellular Ca2+ concentration, and neurotoxicity and increase antioxidant enzyme activities in hippocampal neurons. J Neurochem, 1995. 65(4):p.1740-51.

27. Wang, R. and R.M.D. Holsinger, Exercise-induced brain-derived neurotrophic factor expression: Therapeutic implications for Alzheimer's dementia. Ageing Res Rev, 2018. 48:p.109-121. 